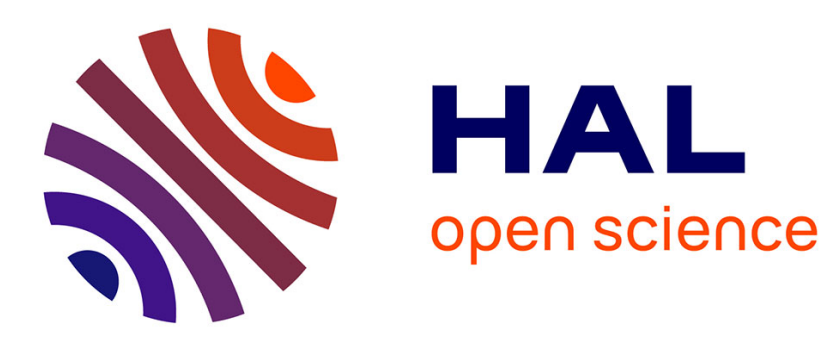

\title{
Experimental investigation of a page-oriented Lippmann "holographic" data storage system
}

\author{
Gilles Pauliat, Kevin Contreras
}

\section{To cite this version:}

Gilles Pauliat, Kevin Contreras. Experimental investigation of a page-oriented Lippmann "holographic" data storage system. Optical Data Storage 2010, May 2010, Boulder, United States. pp.773004. hal-00554909

\section{HAL Id: hal-00554909 \\ https://hal-iogs.archives-ouvertes.fr/hal-00554909}

Submitted on 11 Jan 2011

HAL is a multi-disciplinary open access archive for the deposit and dissemination of scientific research documents, whether they are published or not. The documents may come from teaching and research institutions in France or abroad, or from public or private research centers.
L'archive ouverte pluridisciplinaire HAL, est destinée au dépôt et à la diffusion de documents scientifiques de niveau recherche, publiés ou non, émanant des établissements d'enseignement et de recherche français ou étrangers, des laboratoires publics ou privés. 


\title{
Experimental investigation of a page-oriented Lippmann «holographic» data storage system
}

\author{
Gilles Pauliat, Kevin Contreras \\ Laboratoire Charles Fabry de l'Institut d'Optique, \\ CNRS, Univ Paris-Sud, Campus Polytechnique, RD 128, 91127 Palaiseau cedex, France
}

\begin{abstract}
Lippmann photography is a more than one century old interferometric process invented for recording colored images in thick black and white photographic emulsions. After a comparison between this photographic process and Denisyuk holography, we feature some hints to apply this technique to high density data storage by wavelength multiplexing in a page-oriented approach in thick media. For the first time we experimentally investigate this approach. We anticipated that this storage architecture should allow capacities as large as for conventional holography.
\end{abstract}

Keywords: Optical data storage, holographic and volume memories, Lippmann photography

\section{INTRODUCTION}

The Lippmann interference photographic process ${ }^{1}$ is a technique that shares many features in common with volume holography ${ }^{2,3}$. In Lippmann photography, the image is projected onto a mercury mirror set in close contact with a thick photographic plate. The incident beam interferes with its reflection and records a small Bragg grating inside the thickness of the sensitive layer. At each location of this layer is thus recorded the spectral contents of a pixel of the image. The recorded plate is chemically processed after removing the mirror. Illuminating this processed plate with white light reproduces the coloured image thanks to the Bragg wavelength selectivity. The coloured information of each pixel is thus encoded inside the thickness of the Bragg gratings. The colours are recorded by interferences in a fashion very similar to holographic wavelength multiplexing. The main difference compared to holography is that an additional reference beam is not required. As a consequence of this simplicity, the phase of the incident beam is not recorded: typically, the 3D shape of an object cannot be reproduced. This feature is not a true impediment for data storage. Applying this Lippmann principle to data storage has indeed been proposed for $\operatorname{long}^{4-6}$, but all theses previous demonstrations were implemented in a bit oriented approach. At each location, a focused beam records a series of wavelength multiplexed Bragg gratings. Such stacks of Bragg gratings can be juxtaposed by moving the recording beam at other locations. Because the Rayleigh length of a focused beam scales as the square of its waist radius, such an approach cannot produce high storage densities. Indeed, increasing the number of wavelength multiplexed gratings in a single stack by a factor $p$ requires an increase of the material thickness by the same amount. In order to get a uniform diffraction efficiency in the material thickness, and thus an optimum Bragg selectivity, the Rayleigh length $z_{R}$ must be increased by the same factor $p$. Because pixels must be spaced by about their waist radius $\omega$ to avoid crosstalk, and because this waist radius scales as $\omega \propto \sqrt{z_{R}}$, increasing the number of multiplexed gratings by $p$ results in a decrease of the areal density of multiplexed stacks by $\omega^{2} \propto z_{R} \propto p$. As a net result, and in first approximation, the capacity does not depend of the material thickness.

To circumvent this limitation due to diffraction, it was proposed to confine light in arrays of microfibers. Each stack of microgratings being recording in a microfiber, light confinement prevents diffraction and increasing the material thickness does not decrease the areal density of stacks (i.e. fibers). Consequently, very large capacities should be expected ${ }^{7}$. However, avoiding light coupling from one fiber to the neighbouring ones requires large spacings between fibers, which limits the overall capacity ${ }^{8}$.

As demonstrated in a theoretical previous communication ${ }^{9}$, larger capacities should nevertheless be obtained with a page-oriented approach. Pages are wavelength multiplexed at each location. Simulations ${ }^{9}$ demonstrated that the capacity of such a system should be same as for wavelength multiplexed holographic memories. In the following, we investigate 
such a Lippmann page oriented storage architecture. We first underline the main differences between Denisyuk holography and Lippmann storage and we give some hints for minimizing the inherent image distortion expected for Lippmann storage.

\section{LIPPMANN STORAGE IN THICK MEDIA VERSUS DENISYUK HOLOGRAPHY}

\subsection{Similarities and differences}

In Denisyuk holography, information is recorded by interfering a reference beam, generally a plane wave whose complex amplitude is $R$, with a couterpropagating image beam $A$. This interference pattern imprints a modification of the material refractive index with the modulation term $R^{*} A$. Reading out this information with the very same reference beam $R$ reproduces the original image $R\left(R^{*} A\right) \propto A$. On the contrary, in Lippmann storage, we make interfere the image $A$ with its reflection $A_{R}$. This reflected beam is not a plane wave. To emphasize the difference between Lippmann and Denisyuk storage, we can rewrite $A_{R}$ as $A_{R}=O+\sum_{p \neq 0} a_{p}$ where $O$ is the zero order of the image beam and $\sum_{p \neq 0} a_{p}$ all the other orders. The information is reconstructed with a plane wave corresponding to $O$, so that the reconstructed wavefront is $O\left(O^{*}+\sum_{p \neq 0} a_{p}^{*}\right) A$. The reconstructed wave is no more proportional to $A$ : the presence of all the orders $\sum_{p \neq 0} a_{p}$ should considerably distort the retrieved image.

In a recording medium thinner than the depth of focus of the image, this distortion principally modifies the phase of the retrieved image, without changing its intensity pattern. However, in a thicker medium, at first sight with this simple description, we do not anticipate the correct operation of Lippmann data storage: a very large amount of distortion is expected. This prediction is valid for most images, however, we illustrate with one example in the following paragraph, that for correctly chosen images, the data can be retrieved with a good signal to noise ratio.

\subsection{Simulations}

To illustrate the operation of Lippmann data storage, we rely on computer simulations. Our simulated software was described previously ${ }^{9}$. We assume the material reproduces the interference pattern $A_{R}^{*} A$ under the form of a refractive index modulation $\delta n \propto A_{R}^{*} A$. The pixel pitch of the image to be recorded is $0.8 \mu \mathrm{m}$ so that the image depth of focus is much smaller than the $50 \mu \mathrm{m}$ thick recording material. The image beam is spatially and temporally coherent. The intensity of the image to be recorded is shown in figure 1.a. The pixel amplitudes are binary (0 and 1$)$ and we investigate the influence of their phase contents.

In a first simulation, we selected the phase of these pixel at random between 0 and $2 \pi$. As can be seen in the simulated intensity of the retrieved image, in figure $1 \mathrm{~b}$ ), the original data information is completely blurred and cannot be retrieved.

In figure 1.c, we simulated the retrieved image in case the phase of the original beam is uniform. In such a case, the intensity data can be detected without ambiguity. For sake of comparison, the retrieved image in case of Denisyuk recording is shown in figure 1.d.

A simple explanation can be given for these results. In case the phase is selected at random, the zero order $O$ of the image vanishes and most of the image beam is contained in the other orders: the correlation between wavefront $A_{R}$ and the reference beam used for readout is zero. However in case the phase of the image beam is uniform a large amount of energy is contained in this zero order. Typically, if the sparsity of the recorded data is $50 \%$, then, this zero order contains $50 \%$ of the image energy. The recorded index gratings are thus very close to a Denisyuk hologram and can be readout with the same plane wave.

This illustrates and explains why Lippmann photography can be applied to data storage. As simulated previously, for intensity images such as one used for data storage, Lippmann storage should result in a slight decrease of the diffraction efficiency, but the wavelength selectivity remains identical to the wavelength selectivity of Denisyuk holograms. 


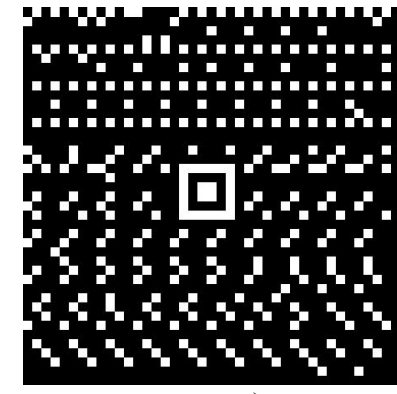

a)

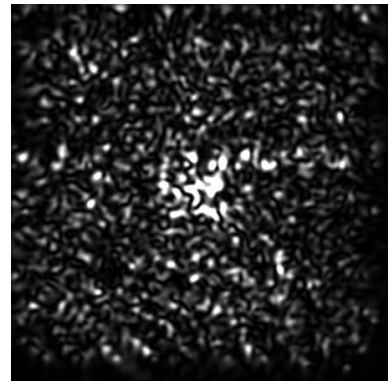

b)

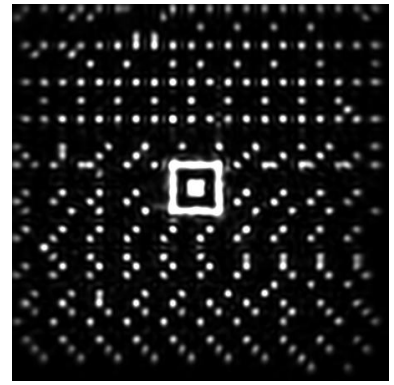

c)

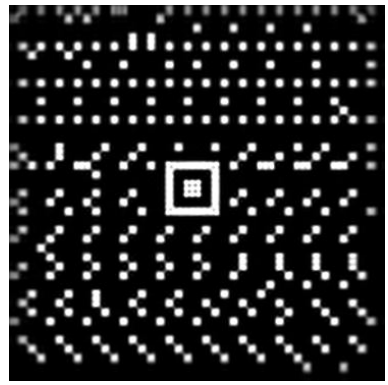

d)

Figure 1. Illustration of noise in Lippmann storage: a) amplitude of the original image before recording; b) simulated intensity of the retrieved image in case the original image contains random phase information; c) simulated intensity of the retrieved image in case the phase of the original image is uniform. The simulated retrieved image in case of a Denisyuk recording is shown in d).

As explained above, the additional term $\sum_{p \neq 0} a_{p}$ is responsible for distortions of the retrieved grey levels: the intensity of one retrieved pixel depends on the value of its neighboring pixels. Lippmann data storage typically produces images with a limited signal to noise ratio. From our simulations ${ }^{9}$, we found that the signal to noise ratio defined by:

$$
S N R=\frac{\mu_{O N}-\mu_{O F F}}{\sqrt{\sigma_{O N}{ }^{2}+\sigma_{O F F}^{2}}}
$$

is about or larger than 4 for binary images with a uniform phase and a sparsity of $50 \%$.

In equation (1), $\mu_{\text {ON/OFF }}, \sigma_{\text {ON/OFF }}$ are the mean values and standard deviations of the retrieved pixel intensities.

This distortion should thus not be the limiting factor in a storage architecture. We checked that the spatial repartition of this distortion is compatible with a block coding scheme ${ }^{10}$.

\section{EXPERIMENTAL SET-UP AND DISCUSSION}

\subsection{Experimental set-up}

The scheme and the picture of our experimental set-up are shown in figure 2. It is fed by a single mode optical fiber in which three lasers are injected: a free running laser diode at $650 \mathrm{~nm}$, a free running diode at $473 \mathrm{~nm}$ and a frequency doubled $\mathrm{Nd}: \mathrm{YVO}_{4}$ laser at $532 \mathrm{~nm}$. Their coherence lengths are much larger than twice the sensitive layer thickness. Shutters allow controlling the exposition durations for each wavelength. The recording material being not sensitive in the $\mathrm{red}$, the wavelength of $650 \mathrm{~nm}$ is used for the alignments only. The other two wavelengths are used for data recording. Light from the fiber illuminates the data mask. We employ a set of chromium masks etched on a glass plate. They are sequentially presented onto the beam by sliding the glass plate in front of the aperture. This mask is then imaged onto the sensitive layer by means of a video lens (focal length $75 \mathrm{~mm}$ ) and a $40 \mathrm{x}$ microscope objective. The correction ring of this microscope objective is adjusted to compensate for the spherical aberration of the thick recording layer and substrate. This recording medium is a thick silver halide holographic plate sensitive at $473 \mathrm{~nm}$ and $532 \mathrm{~nm}$ but not at $650 \mathrm{~nm}$ (Ultimate $08^{11}$ ). This sensitive layer, about $6 \mu \mathrm{m}$ thick, is deposited over a $2 \mathrm{~mm}$ glass substrate. Its thickness has been chosen large enough to produce a Bragg selectivity sufficient to separate the gratings recorded at $473 \mathrm{~nm}$ and $532 \mathrm{~nm}$, but thin enough so that the gratings can be readout at the recording wavelengths in spite of the relatively large shrinkage (about 1\%) of our material during the wet processing. A quarter-wave plate is glued on the substrate surface. The light polarization is chosen so as to direct the diffracted/reflected light through the beam splitter cube according to the arrows shown in figure 2. The images are observed by the CCD camera through a video lens identical to the first one. 

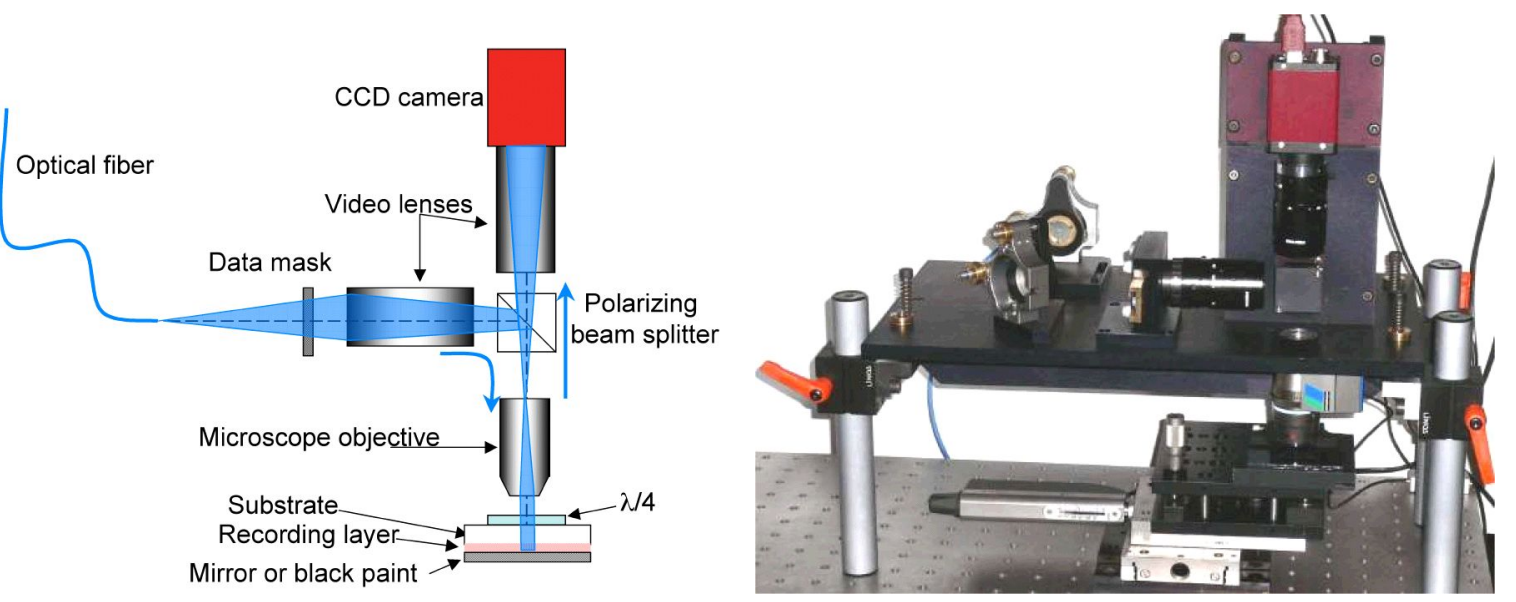

Figure 2. Scheme and photography of the experimental set up.

Using red light, we align the set-up and we image the mask onto the mirror. We then record two images at each location using the two recording wavelengths and two different masks. During this recording, the incident beam interferes with its reflection.

\subsection{Results and discussion}

We investigated two arrangements: this reflected beam either originates from the Fresnel reflection on the interface "air / sensitive layer" (first configuration), or originates from a mirror set below the sensitive layer (second configuration), an index matching liquid between the sensitive layer and mirror prevents further spurious reflections. The material is then processed and a black paint is applied onto the sensitive layer surface. The plate is then repositioned on the set-up to be readout.

Examples of two images superimposed at the same location, with the first configuration, and readout at their recording wavelengths, $532 \mathrm{~nm}$ and $473 \mathrm{~nm}$, are shown in figure 3. No crosstalk is visible in these images.
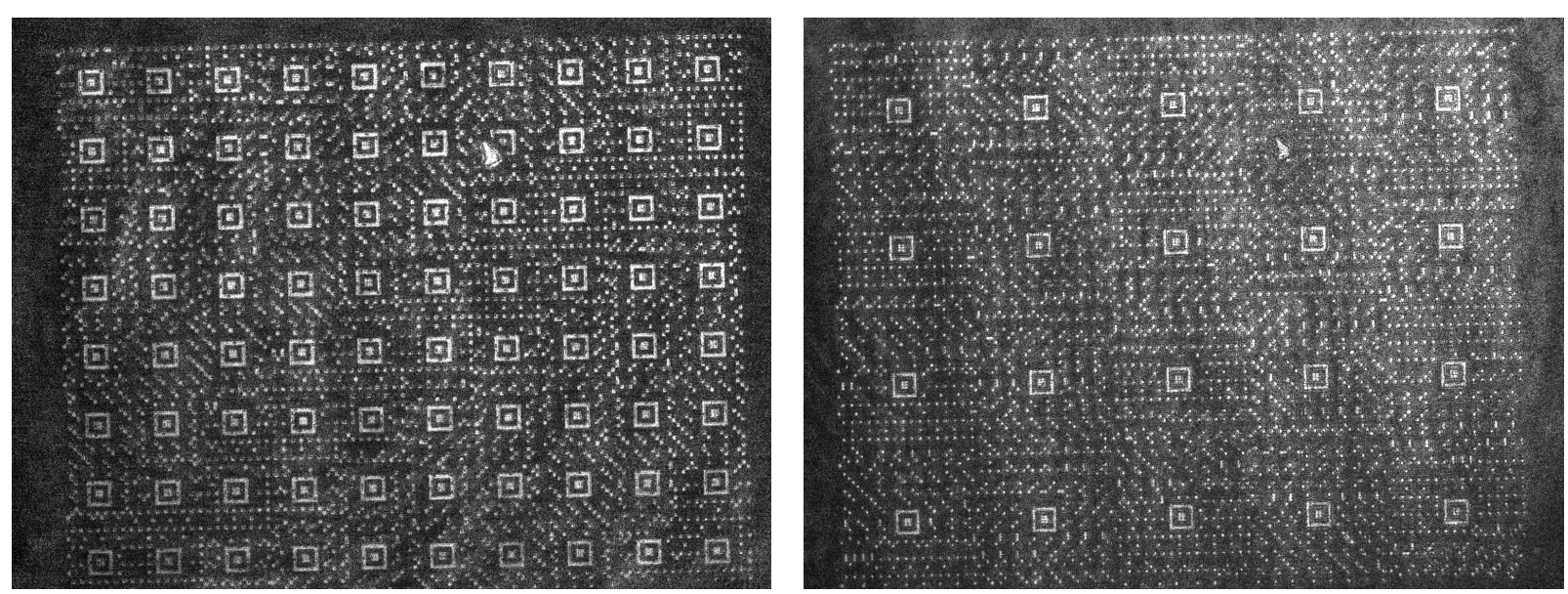

Figure 3. Data pages readout at the same location, a) at $532 \mathrm{~nm}$; b) at $473 \mathrm{~nm}$

The main advantage of the first configuration is to ensure that the bottom interface of the recording layer acts as the Lippmann mirror; no gap exists between the mirror and the recording layer.

For the second configuration, simulations have shown that if a gap is introduced between this mirror and the sensitive layer, then a deterioration of the readout image should happen. 
Comparisons between these two configurations are illustrated on the zooms of different images shown in table 1. First configuration is shown on the first line $(6 \mu \mathrm{m}$ thick emulsion and $0 \mu \mathrm{m}$ gap); the second on the second line $(6 \mu \mathrm{m}$ thick emulsion and $20 \mu \mathrm{m}$ gap); for each configuration we show, from left to right, the original data, the simulated readout, the experimentally reconstructed image. Our results are clearly well described by the simulations: for the $20 \mu \mathrm{m}$ gap, spurious bright spots appear. For a moderately thick medium (as the $6 \mu \mathrm{m}$ used here), it is thus important to minimize the gap in order to reproduce the data correctly.

Table 1. Demonstration of the influence of a gap between the emulsion and the sensitive layer, comparison with simulation.

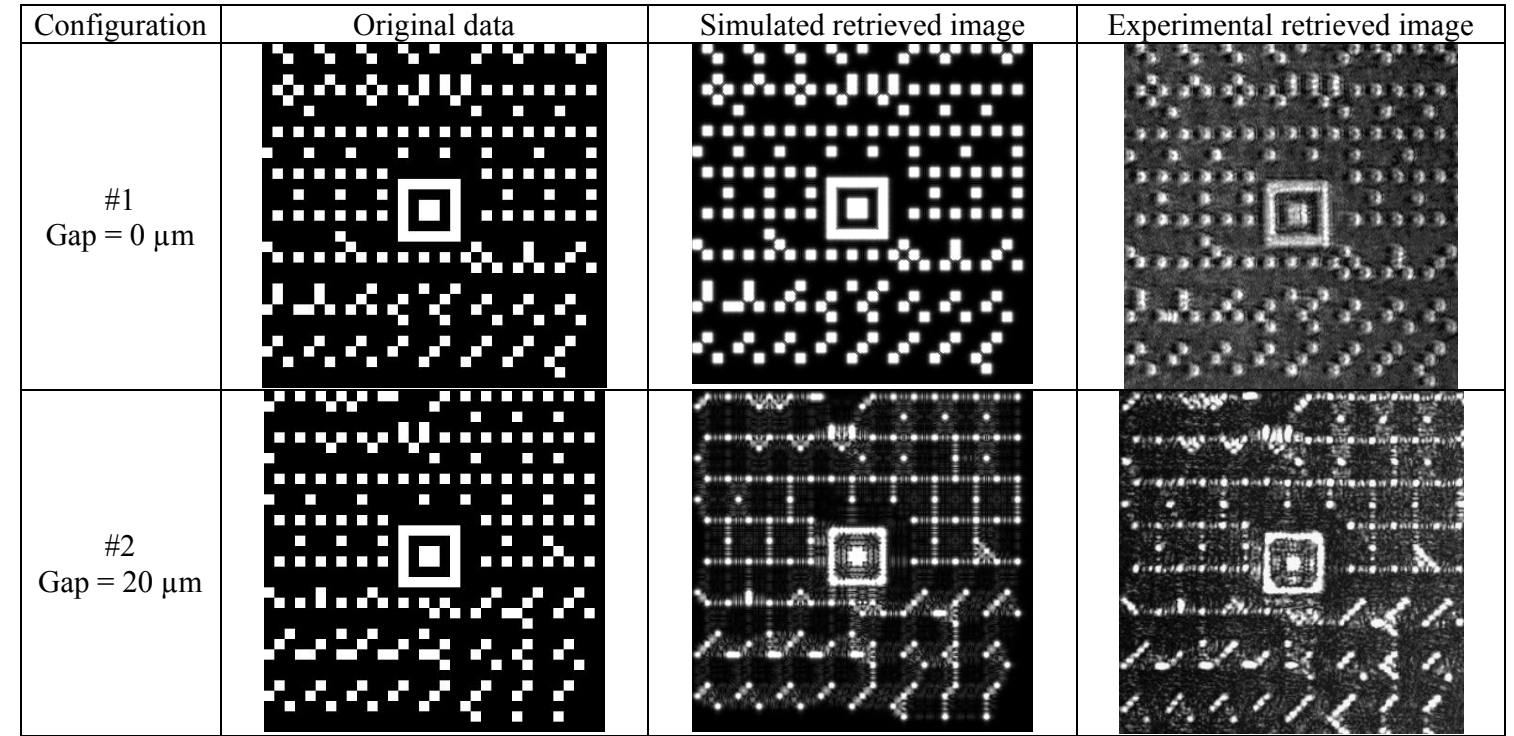

These results validate our simulations. It is worth to note that the same simulations predict that the deterioration introduced by a non zero gap should disappear if the thickness of the recording layer is increased well above the $6 \mu \mathrm{m}$ of our sensitive layer. Current experiments are under progress to check this important feature.

\section{CONCLUSION}

These first experimental results validate our previous theoretical approach. Theory and experiments confirm the potential of Lippmann architectures for reaching very high capacities. The interest in Lippmann architecture is further motivated by the other inherent advantages of Lippmann architectures: very stable and compact interferometric set-up, minimization of the required coherence length, possibility to mass replicate the recorded data by direct copy contact. The absence of an external reference beam allows the image beam to be set perpendicular to the recording medium. This minimizes the optical aberrations and allows using large numerical apertures and thus the largest image resolutions. Lippmann storage does not require the optical access to the two sides of the recording medium. This single side access simplifies the architecture. Furthermore, homodyne detections can be easily implemented to enhanced the detected signals ${ }^{12,13}$.

Acknowledgements: K. Contreras gratefully acknowledges the financial support of the Programme Al $\beta$ an, the European Union Programme of High Level Scholarship for Latin America (E07D401978PE).

\section{REFERENCES}

[1] Lippmann, G., "La photographie des couleurs", C. R. Hebd. Acad. Sci. 112, 274-275 (1891).

[2] Denisyuk, Y.N., "The imaging of the optical properties of an object in a wave field of radiation scattered by it", Opt. Spectrosc. 15, 279-284 (1963). 
[3] Denisyuk, Y.N., "Imaging properties of light intensity waves: the development of the initial Lippmann ideas", J. Optics 22, 275-280 (1991).

[4] Fleisher, H., Pengelly, P., Reynolds, J., Schools, R. and Sincerbox, G., “An optically accessed memory using the Lippmann process for information storage", Opt. and Electro-Opt. Information Processing, MIT Press, 1-30 (1965).

[5] Hoffman, A.S., "Optical information storage in three-dimensional media using the Lippmann Technique", Appl. Opt. 7, 1949-1954 (1968).

[6] Yang, J.J. and Wang, M.R., "White light micrograting multiplexing for high density data storage," Opt. Lett. 31, 1304-1306 (2006).

[7] Labeyrie, A., Huignard, J.P. and Loiseaux, B., "Optical data storage in microfibers", Opt. Lett. 23, 301-303 (1998).

[8] Maire, G., Pauliat, G. and Roosen, G., " Bit-oriented Lippmann and microfiber holographic memories," in Photorefractive Effects, Materials, and Devices, G. Zhang, D. Kip, D. Nolte, and J. Xu, eds., Vol. 99 of OSA Trends in Optics and Photonics (Optical Society of America, 2005), 637-642 (2005).

[9] Contreras, K., Pauliat, G., Arnaud, C. and Roosen, G., "Application of Lippmann interference photography to data storage", J. Europ. Opt. Soc. Rap. Public. 3, 08020 (2008).

[10] Burr, G. W., Ashley, J., Coufal, H., Grygier, R.K., Hoffnagle, J.A.., Jefferson, C.M. and Marcus, B., "Modulation coding for pixel-matched holographic data storage," Opt. Lett. 22, 639-641 (1997).

[11] www.ultimate-holography.com/

[12] Maire, G., Pauliat, G. and Roosen, G., "Homodyne detection readout for bit-oriented holographic memories", Opt. Lett. 31, 175-177 (2006).

[13] Guattari, F., Maire, G., Contreras, K., Arnaud, C., Pauliat, G., Roosen, G., Jradi, S and Carré, C., "Balanced homodyne detection of Bragg microgratings in photopolymer for data storage", Opt. Exp. 5, 2234-2243 (2007). 